Sharif University of Technology
Scientia Iranica
SCIENTIA
IRAN I CA
http://scientiairanica.sharif.edu

\title{
Axial translation of a rigid disc inclusion embedded in a penny-shaped crack in a transversely isotropic solid
}

\author{
S.M. Dehghan Manshadi ${ }^{\mathrm{a}}$, A. Khojasteh ${ }^{\mathrm{b}, *}$, and M. Rahimian ${ }^{\mathrm{a}}$ \\ a. School of Civil Engineering, College of Engineering, University of Tehran, Tehran, P.O. Box 11155-4563, Iran. \\ b. School of Engineering Science, College of Engineering, University of Tehran, Tehran, P.O. Box 11155-4563, Iran. \\ Received 22 December 2016; received in revised form 25 June 2017; accepted 31 July 2017
}

\author{
KEYWORDS \\ Penny-shaped crack; \\ Rigid disc; \\ Transversely isotropic; \\ Axial stiffness; \\ Stress intensity factor; \\ Integral equation.
}

\begin{abstract}
This paper investigates an analytical solution for the axisymmetric interaction of rigid disc inclusion embedded in bonded contact with the surfaces of a penny-shaped crack and a transversely isotropic medium. By using a method of potential functions and treating dual and triple integral equations, the mixed boundary value problem is written in the form of two coupled integral equations, which are amenable to numerical treatments. The axial stiffness of the inclusion and the shearing stress intensity factor at the tip of the penny-shaped crack for different degrees of material anisotropy are illustrated graphically. Useful limiting cases such as a rigid disc inclusion in an uncracked medium and in a completely cracked solid are recovered.
\end{abstract}

(C) 2019 Sharif University of Technology. All rights reserved.

\section{Introduction}

The category of problems that examines the mechanical behavior of contact regions constitutes an important branch of applied mechanics with extensive engineering applications [1]. Nowadays, composites play a very important role in geomechanical engineering. It is common knowledge that all existing structural materials contain different inter- and intra-component defects (cracks, delaminations, etc.) [2]. Analysis of interaction between cracks and inclusion has important applications to the study of micro-mechanics of multiphase materials and to the examination of anchoring devices embedded in geological media [3].

Sneddon [4] considered the distribution of stress produced in the interior of an elastic solid by the

\footnotetext{
*. Corresponding author. Tel.: +9821 61112232;

Fax: +982188078263

E-mail addresses: m.manshadiro@ut.ac.ir (S.M.Dehghan

Manshadi); akhojasteh@ut.ac.ir (A. Khojasteh);

rahimian@ut.ac.ir ( $M$. Rahimian)
}

opening of an internal crack under the action of pressure applied to its surface (see also $[5,6]$ ). By considering various boundary conditions of the crack surfaces, Sneddon and Lowengrub [7] as well as Kassir and Sih [8] obtained associated stress intensity factors. Sih [9] considered the influence of plate thickness on the stress distribution around the crack. Erdogan and Arin [10] investigated the axially symmetric elastostatic problem for a layer bonded to a half-space with different material properties. Rahman [11] investigated dynamic stress intensity factor for a penny-shaped crack embedded in an infinite elastic medium under time harmonic torsional body forces. Selvadurai et al. [12] studied an infinite domain containing a penny-shaped crack, which is loaded axially by a rigid disc inclusion in an isotropic elastic medium. Selvadurai [3] performed analysis of the problem pertinent to the complete indentation of a single face of a penny-shaped crack by a rigid smooth inclusion. The problem of examining the axial tensile loading of a rigid circular disc, bonded to the surface of a half-space weakened by a penny-shaped crack, was considered by Selvadurai [1]. Selvadurai [13] 
studied the axial loading of an annular crack by a rigid disc inclusion and presented the shearing stress intensity factor and the axial stiffness of the inclusion for different ratios of the inner and outer radii of the annular crack. Vrbik et al. [14] studied the symmetric indentation of a penny-shaped crack by a smoothly embedded rigid circular disc inclusion in a thick layer.

Recently, Eskandari et al. [15] discussed separation of dissimilar piezoelectric half-spaces by a rigid disc inclusion. Shodja et al. [16] analyzed the interaction of the annular and penny-shaped cracks in an infinite piezoelectric medium (see also [17]). Singh et al. [18] performed the study of indentation of an elliptic crack by a rigid elliptic inclusion in the anti-plane shear mode. Eskandari-Ghadi et al. [19] presented a mathematical formulation for the analysis of a transversely isotropic half-space containing a discshaped crack buried at an arbitrary depth. Fabrikant [20] considered a transversely isotropic body weakened on the plane perpendicular to the planes of isotropy of the transversely isotropic body (see also [2123]). Yang and Zhao [24] investigated the influence of a capillary bridge or a liquid droplet on the crack opening and stress intensity factor of a penny-shaped crack under a far-field tensile stress. Shahmohamadi et al. [25] studied the axial interaction of a rigid disc with a penny-shaped crack in a transversely isotropic full-space. Moreover, Antipov and Mkhitaryan [26] studied the plane problem of interaction between a thin rigid inclusion and a finite crack. Amiri-Hezaveh et al. [27] examined the dynamic indentation of a rigid circular plate embedded in a non-homogeneous transversely isotropic full-space. Eskandari-Ghadi et al. [28] presented an analytical solution for a two-layer transversely isotropic half-space containing a pennyshaped crack located at the interface of layers.

In this paper, the main objective is to investigate the interaction of a rigid disc in bonded contact with the surfaces of a penny-shaped crack and an infinite transversely isotropic medium. By virtue of appropriate displacement-potential functions and Hankel and Abel transforms, the solution of the problem is reduced to two coupled Fredholm integral equations, which are amenable to numerical treatments. The axial stiffness of the inclusion and the mode II stress intensity factor at the tip of the crack are obtained for several types of hypothetical transversely isotropic materials.

From a practical viewpoint, in geomechanical applications, the rigid disc-shaped inclusion represents the behavior of an earth or rock anchor that is created by the hydraulic fracture of the earth or rock mass. The inclusion represents the resinous or cementing material that is used to transfer anchoring loads to the geological medium [29].

\section{Statement of the problem and the governing equations}

Consider a rigid circular disc inclusion of radius $a$ surrounded by a penny-shaped crack of radius $b$ in an infinite transversely isotropic medium while the disc is in perfect contact with the surfaces of the crack (see Figure 1). The disc is subjected to a central force $T$ which induces a rigid-body displacement $\delta$ in $z$ direction. Because of symmetry, it suffices to limit attention to one half-space $(0 \leq z<\infty)$. The mixed boundary conditions of the problem under consideration in terms of displacement vector $\boldsymbol{u}$ and Cauchy stress tensor $\boldsymbol{\sigma}$ are as follows:

$$
\begin{array}{ll}
u_{z}(r, 0)=\delta, & 0 \leq r \leq a, \\
u_{r}(r, 0)=0, & 0 \leq r \leq a, \quad b \leq r<\infty, \\
\sigma_{z z}(r, 0)=0, & a<r<\infty, \\
\sigma_{r z}(r, 0)=0, & a<r<b .
\end{array}
$$

For axisymmetric problems, the equilibrium equations of the static motion for a homogeneous transversely isotropic elastic solid in terms of displacement and in the absence of the body forces can be expressed as follows [30]:

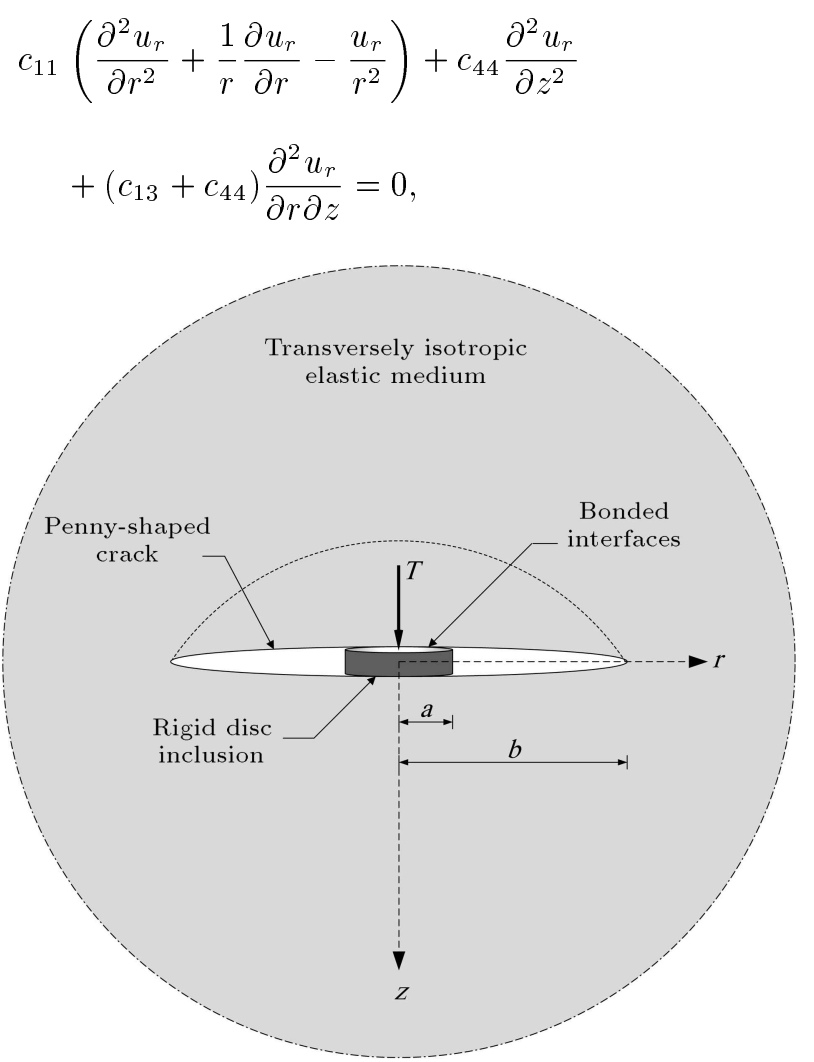

Figure 1. Axial translation of a rigid disc inclusion embedded in a penny-shaped crack. 


$$
\begin{gathered}
c_{44}\left(\frac{\partial^{2} u_{z}}{\partial r^{2}}+\frac{1}{r} \frac{\partial u_{z}}{\partial r}\right)+c_{33} \frac{\partial^{2} u_{z}}{\partial z^{2}} \\
+\left(c_{13}+c_{44}\right)\left(\frac{\partial^{2} u_{r}}{\partial r \partial z}+\frac{1}{r} \frac{\partial u_{r}}{\partial z}\right)=0,
\end{gathered}
$$

where $u_{r}$ and $u_{z}$ are the displacement components in $r$ and $z$ directions, respectively, and $c_{i j}$ represents the elastic constants of the solid. The displacement and stress fields for a semi-infinite transversely isotropic medium $(0 \leq z<\infty)$ are as follows [31]:

$$
\begin{aligned}
u_{z}(r, z)= & \int_{0}^{\infty}-\xi^{3}\left[A^{\prime}(\xi)\left(1+\alpha_{1}-s_{1}^{2} \alpha_{2}\right) \mathrm{e}^{-\xi s_{1} z}\right. \\
& \left.+B^{\prime}(\xi)\left(1+\alpha_{1}-s_{2}^{2} \alpha_{2}\right) \mathrm{e}^{-\xi s_{2} z}\right] J_{0}(\xi r), \mathrm{d} \xi \\
u_{r}(r, z)= & \int_{0}^{\infty}-\xi^{3} \alpha_{3}\left[A^{\prime}(\xi) s_{1} \mathrm{e}^{-\xi s_{1} z}\right. \\
& \left.+B^{\prime}(\xi) s_{2} \mathrm{e}^{-\xi s_{2} z}\right] J_{1}(\xi r) \mathrm{d} \xi \\
\sigma_{z z}(r, z)= & \int_{0}^{\infty}-\xi^{4}\left(A^{\prime}(\xi) s_{1} \mathrm{e}^{-\xi s_{1} z}+B^{\prime}(\xi) s_{2} \mathrm{e}^{-\xi s_{2} z}\right) \\
& {\left[c_{33} \alpha_{2}\left(s_{1}^{2}+s_{2}^{2}\right)+\left(c_{13} \alpha_{3}\right.\right.} \\
& \left.\left.-c_{33}\left(1+\alpha_{1}\right)\right)\right] J_{0}(\xi r) \mathrm{d} \xi, \\
& \mathrm{e}^{-\xi s_{2} z}+J_{1}(\xi r) \mathrm{d} \xi, \\
\sigma_{r z}(r, z)= & \int_{0}^{\infty} \xi^{4} c_{44}\left[A^{\prime}(\xi)\left(1+\alpha_{1}+s_{1}^{2}\left(\alpha_{3}-\alpha_{2}\right)\right)\right. \\
& \\
& \\
&
\end{aligned}
$$

where $A^{\prime}(\xi)$ and $B^{\prime}(\xi)$ are unknown functions and

$$
\alpha_{1}=\frac{c_{12}+c_{66}}{c_{66}}, \quad \alpha_{2}=\frac{c_{44}}{c_{66}}, \quad \alpha_{3}=\frac{c_{13}+c_{44}}{c_{66}} .
$$

Herein, $s_{1}$ and $s_{2}$ are the roots of the following equation that, in view of the positive-definiteness of the strain energy, are not zero or pure imaginary numbers [30]:

$$
c_{33} c_{44} s^{4}-\left[c_{13}^{2}+2 c_{13} c_{44}-c_{11} c_{33}\right] s^{2}+c_{11} c_{44}=0 .
$$

Introducing the substitutions:

$$
[A(\xi) ; B(\xi)]=\frac{1}{\xi^{2}}\left[A^{\prime}(\xi) ; B^{\prime}(\xi)\right]
$$

and using Eq. (6), boundary conditions (1)-(4) can be reduced to the following system of integral equations:

$$
\begin{aligned}
& \int_{0}^{\infty} M(\xi) J_{0}(\xi r) \mathrm{d} \xi=\delta, \quad 0 \leq r \leq a \\
& \int_{0}^{\infty} N(\xi) J_{1}(\xi r) \mathrm{d} \xi=\gamma_{1} \int_{0}^{\infty} M(\xi) J_{1}(\xi r) \mathrm{d} \xi \\
& 0 \leq r \leq a, \quad b \leq r<\infty \\
& \int_{0}^{\infty} \xi M(\xi) J_{0}(\xi r) \mathrm{d} \xi=\gamma_{2} \int_{0}^{\infty} \xi N(\xi) J_{0}(\xi r) \mathrm{d} \xi \\
& a<r<\infty, \quad a<r<b, \\
& \int_{0}^{\infty} \xi N(\xi) J_{1}(\xi r) \mathrm{d} \xi=0, \quad a
\end{aligned}
$$

where $\gamma_{k}(k=1,2)$ and functions $M(\xi)$ and $N(\xi)$ are mentioned in Appendix A.

\subsection{Dual integral equations}

By making use of Eqs. (10) and (12) the following system of dual integral equations is obtained:

$$
\begin{aligned}
& \int_{0}^{\infty} M(\xi) J_{0}(\xi r) \mathrm{d} \xi=\delta, \quad 0 \leq r \leq a \\
& \int_{0}^{\infty} \xi M(\xi) J_{0}(\xi r) \mathrm{d} \xi=\gamma_{2} \int_{0}^{\infty} \xi N(\xi) J_{0}(\xi r) \mathrm{d} \xi, \\
& \quad a<r<\infty .
\end{aligned}
$$

The integral equations (14) and (15) yield the following:

$$
M(\xi)=\frac{2}{\pi}\left[\frac{\delta \sin (\xi a)}{\xi}+\int_{a}^{\infty} F(u) \cos (\xi u) \mathrm{d} u\right],
$$

where:

$$
F(t)=\gamma_{2} \int_{0}^{\infty} N(\xi) \cos (\xi t) \mathrm{d} \xi
$$

\subsection{Triple integral equations}

By making use of Eqs. (11) and (13), the following system of triple integral equations is obtained:

$$
\begin{aligned}
& \int_{0}^{\infty} N(\xi) J_{1}(\xi r) \mathrm{d} \xi=\gamma_{1} \int_{0}^{\infty} M(\xi) J_{1}(\xi r) \mathrm{d} \xi \\
& 0 \leq r \leq a, \\
& \int_{0}^{\infty} \xi N(\xi) J_{1}(\xi r) \mathrm{d} \xi=0, \quad a<r<b, \\
& \int_{0}^{\infty} N(\xi) J_{1}(\xi r) \mathrm{d} \xi=\gamma_{1} \int_{0}^{\infty} M(\xi) J_{1}(\xi r) \mathrm{d} \xi, \\
& b \leq r<\infty .
\end{aligned}
$$

Taking the following assumption: 


$$
\int_{0}^{\infty} \xi N(\xi) J_{1}(\xi r) \mathrm{d} \xi= \begin{cases}f_{1}(r), & 0<r<a \\ 0, & a<r<b \\ f_{2}(r), & b<r<\infty\end{cases}
$$

By employing the inverse Hankel integral transform to Eq. (21), the following relation is obtained:

$$
N(\xi)=\int_{0}^{a} r f_{1}(r) J_{1}(\xi r) \mathrm{d} r+\int_{b}^{\infty} r f_{2}(r) J_{1}(\xi r) \mathrm{d} r
$$

Inserting Eq. (22) into Eq. (11), we have:

$$
\begin{array}{ll}
I_{1}(r)+I_{2}(r)=g(r), & 0<r<a, \\
I_{1}(r)+I_{2}(r)=g(r), & b<r<\infty
\end{array}
$$

where:

$$
\begin{aligned}
& I_{j}(r)=\int \lambda f_{j}(\lambda) L(r, \lambda) \mathrm{d} \lambda, \quad(j=1,2), \\
& L(r, \lambda)=\int_{0}^{\infty} J_{1}(\xi r) J_{1}(\xi \lambda) \mathrm{d} \xi, \\
& g(r)=\gamma_{1} \int_{0}^{\infty} M(\xi) J_{1}(\xi r) \mathrm{d} \xi,
\end{aligned}
$$

and the limits of integration in Eq. (25) can occupy $(0, a)$ and $(b, \infty)$ ranges depending upon the value of $j$.

Using the procedure presented in [32], Eqs. (23) and (24) can be rewritten as follows:

$$
\begin{aligned}
& \int_{0}^{r} \frac{F_{1}(s) \mathrm{d} s}{\left(r^{2}-s^{2}\right)^{1 / 2}}=-r^{2} \int_{b}^{\infty} \frac{F_{2}(s) \mathrm{d} s}{s^{2}\left(s^{2}-r^{2}\right)^{1 / 2}}+\frac{\pi r}{2} g(r), \\
& 0<r<a, \\
& \int_{r}^{\infty} \frac{F_{2}(s) \mathrm{d} s}{s^{2}\left(s^{2}-r^{2}\right)^{1 / 2}}=-\frac{1}{r^{2}} \int_{0}^{a} \frac{F_{1}(s) \mathrm{d} s}{\left(r^{2}-s^{2}\right)^{1 / 2}}+\frac{\pi}{2 r} g(r), \\
& b<r<\infty
\end{aligned}
$$

where:

$$
\begin{aligned}
& F_{1}(s)=s^{2} \int_{s}^{a} \frac{f_{1}(u) \mathrm{d} u}{\left(u^{2}-s^{2}\right)^{1 / 2}} \\
& F_{2}(s)=\int_{b}^{s} \frac{u^{2} f_{2}(u) \mathrm{d} u}{\left(s^{2}-u^{2}\right)^{1 / 2}}
\end{aligned}
$$

Eqs. (28) and (29) are the Abel type integral equations, whose solutions are as follows:

$$
\begin{aligned}
F_{1}(s)= & -\frac{2}{\pi} \int_{b}^{\infty}\left[-\frac{s^{2}}{u\left(s^{2}-u^{2}\right)}+\frac{s}{2 u^{2}} \ln \left|\frac{s+u}{s-u}\right|\right] \\
& F_{2}(u) \mathrm{d} u+\frac{\mathrm{d}}{\mathrm{d} s} \int_{0}^{s} \frac{r^{2} g(r) \mathrm{d} r}{\left(s^{2}-r^{2}\right)^{1 / 2}}, \\
0< & s<a, \\
F_{2}(s)= & -s^{2} \frac{\mathrm{d}}{\mathrm{d} s} \int_{s}^{\infty} \frac{g(r) \mathrm{d} r}{\left(r^{2}-s^{2}\right)^{1 / 2}} \\
& -\frac{2}{\pi} \int_{0}^{a}\left[\frac{s}{s^{2}-u^{2}}+\frac{1}{2 u} \ln \left|\frac{s+u}{s-u}\right|\right] F_{1}(u) \mathrm{d} u \\
b<s< & \infty .
\end{aligned}
$$

Using the following relations:

$$
\begin{aligned}
& \frac{\mathrm{d}}{\mathrm{d} s} \int_{0}^{s} \frac{r^{2} J_{1}(\xi r) \mathrm{d} r}{\sqrt{s^{2}-r^{2}}}=s \sin (\xi s), \\
& \frac{\mathrm{d}}{\mathrm{d} s} \int_{s}^{\infty} \frac{J_{1}(\xi r) \mathrm{d} r}{\sqrt{r^{2}-s^{2}}}=\frac{\cos (\xi s)}{s}-\frac{\sin (\xi s)}{\xi s^{2}},
\end{aligned}
$$

and Eq. (27), we have:

$$
\begin{aligned}
& \frac{\mathrm{d}}{\mathrm{d} s} \int_{0}^{s} \frac{r^{2} g(r) \mathrm{d} r}{\left(s^{2}-r^{2}\right)^{1 / 2}}=\gamma_{1} s \int_{0}^{\infty} M(\xi) \sin (\xi s) \mathrm{d} \xi \\
& \quad 0<s<a, \\
& s^{2} \frac{\mathrm{d}}{\mathrm{d} s} \int_{s}^{\infty} \frac{g(r) \mathrm{d} r}{\left(r^{2}-s^{2}\right)^{1 / 2}}=\gamma_{1} \int_{0}^{\infty} \frac{1}{\xi}[\xi s \cos (\xi s) \\
& \quad-\sin (\xi s)] M(\xi) \mathrm{d} \xi, \quad b<s<\infty .
\end{aligned}
$$

Insertion of Eqs. (36) and (37) into Eqs. (32) and (33) yields the following coupled Fredholm integral equations:

$$
\begin{aligned}
F_{1}(s) & -\frac{2}{\pi^{2}} \gamma_{1} \gamma_{2} \int_{0}^{a} \frac{s F_{1}(u)}{u\left(u^{2}-s^{2}\right)}\left[u \ln \left|\frac{a-s}{a+s}\right|\right. \\
& \left.-s \ln \left|\frac{a-u}{a+u}\right|\right] \mathrm{d} u+\frac{2}{\pi} \int_{b}^{\infty}\left[-\frac{s^{2}}{u\left(s^{2}-u^{2}\right)}\right. \\
& \left.+\frac{s}{2 u^{2}} \ln \left|\frac{s+u}{s-u}\right|\right] F_{2}(u) \mathrm{d} u \\
& +\frac{2}{\pi} \gamma_{1} \gamma_{2} \int_{b}^{\infty}\left[\frac{s}{2 u^{2}} \ln \left|\frac{(u-s)(a+s)}{(u+s)(a-s)}\right|\right. \\
& \left.-\frac{s^{2}}{u\left(u^{2}-s^{2}\right)}\right] F_{2}(u) \mathrm{d} u=\frac{\gamma_{1} \delta}{\pi} s \ln \left|\frac{s+a}{s-a}\right|,
\end{aligned}
$$

$0<s<a$, 


$$
\begin{gathered}
\left(1-\gamma_{1} \gamma_{2}\right) F_{2}(s)+\frac{2}{\pi} \int_{0}^{a}\left[\frac{s}{s^{2}-u^{2}}+\frac{1}{2 u} \ln \left|\frac{s+u}{s-u}\right|\right] \\
F_{1}(u) \mathrm{d} u-\frac{2}{\pi} \gamma_{1} \gamma_{2} \int_{0}^{a} \frac{s F_{1}(u)}{s^{2}-u^{2}} \mathrm{~d} u \\
+\gamma_{1} \gamma_{2} a \int_{c}^{\infty} \frac{F_{2}(u)}{u^{2}} \mathrm{~d} u \\
+\frac{\gamma_{1} \gamma_{2}}{\pi} \int_{0}^{a} \frac{F_{1}(u)}{u} \ln \left|\frac{(s-u)(a+u)}{(s+u)(a-u)}\right| \mathrm{d} u \\
=\gamma_{1} a \delta, \quad b<s<\infty .
\end{gathered}
$$

We can rewrite Eqs. (38) and (39) in the general forms:

$$
\begin{aligned}
\phi_{1}(s) & +\int_{0}^{a} \phi_{1}(u) K_{11}(u, s) \mathrm{d} u \\
& +\int_{b}^{\infty} \phi_{2}(u) K_{12}(u, s) \mathrm{d} u=L_{1}(s) \delta, \\
0<s<a & \\
R \phi_{2}(s) & +\int_{0}^{a} \phi_{1}(u) K_{21}(u, s) \mathrm{d} u \\
& +\int_{b}^{\infty} \phi_{2}(u) K_{22}(u, s) \mathrm{d} u=L_{2}(s) \delta, \\
b< & <\infty
\end{aligned}
$$

where we have assumed that $F_{1}(s)$ and $F_{2}(s)$ admit representations of the form:

$$
\left[F_{1}(u) ; F_{2}(u)\right]=\frac{\gamma_{1} u}{\pi}\left[\phi_{1}(u) ; \phi_{2}(u)\right],
$$

and kernel functions $K_{i j}(i, j=1,2)$ are expressed as follows:

$$
\begin{aligned}
K_{11}(u, s)= & \frac{\eta_{1}}{u^{2}-s^{2}}\left(u \ln \left|\frac{a-s}{a+s}\right|-s \ln \left|\frac{a-u}{a+u}\right|\right), \\
K_{22}(u, s)= & \eta_{2} \frac{a}{s u}, \\
K_{12}(u, s)= & \eta_{3}\left(-\frac{s}{s^{2}-u^{2}}+\frac{1}{2 u} \ln \left|\frac{s+u}{s-u}\right|\right) \\
& +\eta_{4} \frac{1}{2 u} \ln \left|\frac{a+s}{a-s}\right|, \\
K_{21}(u, s)= & \eta_{3}\left(-\frac{u}{u^{2}-s^{2}}+\frac{1}{2 s} \ln \left|\frac{u+s}{u-s}\right|\right) \\
& +\eta_{4} \frac{1}{2 s} \ln \left|\frac{a+u}{a-u}\right| .
\end{aligned}
$$

Constants $R$ and $\eta_{k}(k=1, \ldots, 4)$ are given by:

$$
\begin{aligned}
& R=1-\gamma_{1} \gamma_{2}, \\
& \eta_{1}=-\frac{2}{\pi^{2}} \gamma_{1} \gamma_{2}, \quad \eta_{2}=\gamma_{1} \gamma_{2}, \\
& \eta_{3}=\frac{2}{\pi}\left(1-\gamma_{1} \gamma_{2}\right), \quad \eta_{4}=\frac{2}{\pi} \gamma_{1} \gamma_{2},
\end{aligned}
$$

and functions $L_{1}(s)$ and $L_{2}(s)$ are defined by:

$$
L_{1}(s)=\ln \left|\frac{s+a}{s-a}\right|, \quad L_{2}(s)=\frac{\pi a}{s} .
$$

\section{Contact-load distribution and axial stiffness}

A practical interesting result is the load-displacement relationship. The resultant force, $T$, acting on the disc is calculated by:

$$
T=2 \int_{0}^{2 \pi} \int_{0}^{a} r \sigma_{z z}(r, 0) \mathrm{d} r \mathrm{~d} \theta
$$

where the axial stress, $\sigma_{z z}$, in the inclusion region is given by:

$$
\begin{aligned}
\sigma_{z z}(r, 0)= & \int_{0}^{\infty} \xi M(\xi) J_{0}(\xi r) \mathrm{d} \xi \\
& -\gamma_{2} \int_{0}^{\infty} \xi N(\xi) J_{0}(\xi r) \mathrm{d} \xi, \quad 0<r<a .
\end{aligned}
$$

By employing the identities $M(\xi)$ and $N(\xi)$ as defined in Eqs. (16) and (22), Eq. (46) simplifies to:

$$
\begin{aligned}
T= & 8 a \eta_{4}+\frac{8 \gamma_{1} \eta_{3}}{\pi^{2}}\left[\int_{0}^{a} \phi_{1}(u) \ln \left|\frac{a+u}{a-u}\right| \mathrm{d} u\right. \\
& \left.+\pi a \int_{b}^{\infty} \frac{\phi_{2}(u)}{u} \mathrm{~d} u\right] .
\end{aligned}
$$

\section{Stress intensity factor at the crack tip}

A quantity of physical interest, which is applicable in fracture mechanics, is the stress intensity factor. Due to asymmetric deformation about $z=0$, the only non-zero stress component is $\sigma_{r z}$. The mode II stress intensity factor is defined by:

$$
K_{I I}^{b}=\lim _{r \rightarrow b^{+}}[2(r-b)]^{1 / 2} \sigma_{r z}(r, 0),
$$

where:

$$
\sigma_{r z}(r, 0)=\int_{0}^{\infty} \xi N(\xi) J_{1}(\xi r) \mathrm{d} \xi, \quad r>b .
$$

Utilizing Eq. (21), we find that:

$$
\begin{aligned}
& \sigma_{r z}(r, 0)=\frac{2 F_{2}(b)}{\pi r\left(r^{2}-b^{2}\right)^{1 / 2}}+\frac{2}{\pi r} \int_{b}^{r} \frac{F_{2}^{\prime}(s) \mathrm{d} s}{\left(r^{2}-s^{2}\right)^{1 / 2}} . \\
& r>b .
\end{aligned}
$$

Inserting Eqs. (42) and (51) into Eq. (49), the mode II stress intensity factor can be expressed as follows: 


$$
K_{I I}^{b}=\frac{2 \gamma_{1} \phi_{2}(b)}{\pi^{2} \sqrt{b}}
$$

\section{Special cases}

Before proceeding to the numerical solution of the general problem, it is relevant to examine some limiting cases whose solutions are available. Herein, five special cases are inferred: (i) intact medium; (ii) completely cracked solid; (iii) direct loading of a penny-shaped crack; (iv) $s_{2} \rightarrow s_{1}$; and (v) effect of incompressible materials.

\subsection{Inclusion in an uncracked elastic solid full-space}

The force $T$ required to achieve the disc displacement $\delta$ in $z$ direction is equal to the following (see Figure 2(a)):

$$
T=\frac{8 c_{44} c_{33} a \delta\left(s_{1}+s_{2}\right)}{c_{44}+\sqrt{c_{11} c_{33}}},
$$

where a transversely isotropic medium [33] and an isotropic medium simplifies to [34] are simplified to:

$$
T=\frac{32 G a \delta(1-\nu)}{3-4 \nu}
$$

in which $G$ and $\nu$ are the elastic shear modulus and Poisson's ratio for an isotropic medium, respectively.

\subsection{Disc inclusion embedded between two half-space regions}

Considering the limit $a / b \rightarrow 0$ (with $a \neq 0$ ), the problem turns into the disc inclusion which is embedded between two identical half-space regions (see Figure 2(b)). In this case, the total force is:

$$
T=\frac{2 a \theta \delta}{H \tanh (\pi \theta)},
$$

where $H$ and $\theta$ are mentioned in Appendix B. This relation, corresponding to a transversely isotropic medium as reported by Fabrikant [35] and for an isotropic medium, simplifies to [36]:

$$
T=\frac{8 G a \delta \ln (3-4 \nu)}{1-2 \nu} .
$$

\subsection{Direct loading of a penny-shaped crack}

The exact mode II stress intensity factor of a pennyshaped crack in an infinite transversely isotropic medium due to a axial point force parallel to the $z$ axis applied at the center of the crack is obtained as follows [37]:

$$
K_{I I}^{b}=\frac{T}{2 \pi b^{3 / 2}}\left(\frac{m_{1} s_{1}}{m_{1}-1}+\frac{m_{2} s_{2}}{m_{2}-1}\right),
$$

where $m_{k}(k=1,2)$ is defined in Appendix B, which, for the isotropic case, simplifies to [8]:

$$
K_{I I}^{b}=\frac{T(1-2 \nu)}{8 \pi(1-\nu) b^{3 / 2}} .
$$

In this study, as $a \rightarrow 0$ (see Figure $2(\mathrm{c})$ ), implying the inclusion disappears, parameter $T$ in Eqs. (57) and (58) can be obtained from Eqs. (55) and (56), respectively.

\section{4. $s_{1}$ and $s_{2}$ become equal}

By substituting $s_{1}=s_{2}$ into Eq. (6), terms with forms of $0 / 0$ will be encountered. This occurs in transversely isotropic materials when $\sqrt{c_{11} c_{33}}-c_{13}-2 c_{44}=0$. In this case, one can obtain $s_{1}=s_{2}=\left(c_{11} / c_{33}\right)^{1 / 4}$. Therefore, in order to obtain displacement and stress potential relations of the case of $s_{1}=s_{2}$, it is required to take the limits by setting $s_{2} \rightarrow s_{1}$. The results are presented in Appendix C.

\subsection{Material incompressibility effect in an isotropic medium}

In the limiting case of material incompressibility $(\nu=$ $0.5)$, Eqs. (54) and (56) reduce to the same result as follows:

$$
T=16 G a \delta \text {. }
$$

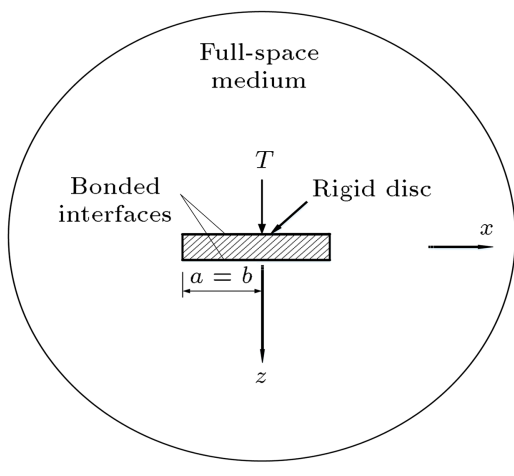

(a)

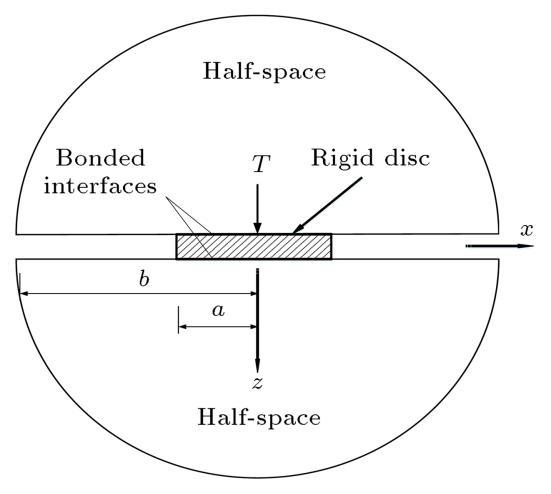

(b)

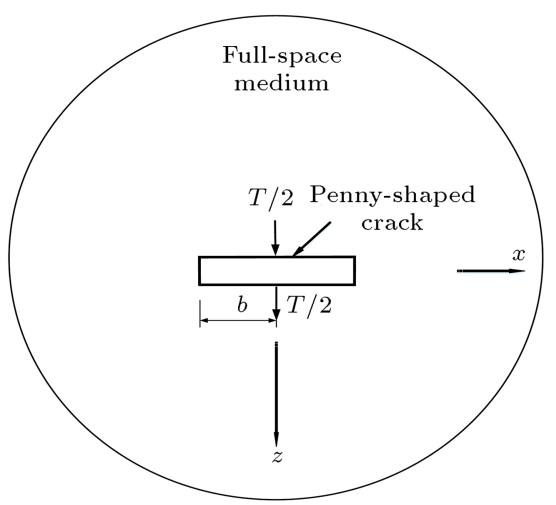

(c)

Figure 2. Axial translation of a rigid disc inclusion located on a cracked plane: Limiting cases: (a) An intact full-space medium, (b) two half-space regions, and (c) direct loading of a penny-shaped crack. 
From the above relation, it is evident that, in the incompressible elastic materials, the extent of cracking and an increase in the value of $b$, on the plane containing the rigid inclusion, have no effect on the axial stiffness of the inclusion (see [38]).

\section{The numerical evaluation of the governing integral equations}

The simultaneous coupled Fredholm integral equations of the second kind (40) and (41) governing the axisymmetric interaction of a penny-shaped crack and a rigid disc inclusion are not amenable to solution in an exact form. A variety techniques was proposed for the numerical solution of coupled systems of Fredholm integral equations of the general type described by Baker [39] and Atkinson [40].

To solve these coupled integral equations numerically, integration intervals $(0, a)$ and $(b, \infty)$ are divided to $N_{1}, N_{2}$ segments, respectively, and end points of the segments can be expressed as follows:

$$
\begin{aligned}
& x_{i}=(2 i-1) h_{1} \quad \text { with } i=1,2, \ldots, N_{1}, \\
& t_{i}=t_{i-1}+\alpha\left(t_{i-1}+t_{i-2}\right), \\
& \text { with } i=3,4, \ldots, N_{2}+1,
\end{aligned}
$$

where $h_{1}=a / 2 N_{1}, t_{1}=b, t_{2}=b+a / N_{1}$, and $\alpha$ is a constant of proportionality such that the interval $(b, \infty)$ is approximated in the numerical scheme. For the treatment of coupled integral equations (Eqs. (40) and (41)), according to standard quadrature method, the integral equations can be written in the discretized form as:

$$
\left[\begin{array}{cc}
D_{1} & D_{2} \\
D_{3} & D_{4}
\end{array}\right]\left\{\begin{array}{l}
\phi_{1}(s) \\
\phi_{2}(s)
\end{array}\right\}=\left\{\begin{array}{l}
L_{1}(s) \\
L_{2}(s)
\end{array}\right\}
$$

where:

$$
\begin{aligned}
D_{1}= & \delta_{i j}+\sum_{i=1}^{N_{1}} \sum_{j=1}^{N_{1}} \frac{w_{j} \eta_{1}}{u_{j}^{2}-s_{i}^{2}}\left[u_{j} \ln \left|\frac{a-s_{i}}{a+s_{i}}\right|\right. \\
& \left.-s_{i} \ln \left|\frac{a-u_{j}}{a+u_{j}}\right|\right], \quad i \neq j \\
D_{1}= & \delta_{i i}+\sum_{i=1}^{N_{1}} \frac{w_{i} \eta_{1}}{2 s_{i}}\left[\frac{2 a s_{i}}{a^{2}-s_{i}^{2}}+\ln \left|\frac{a-s_{i}}{a+s_{i}}\right|\right] \\
& i=j, \\
D_{2}= & \sum_{i=1}^{N_{1}} \sum_{j=1}^{N_{2}} w_{j} \eta_{3}\left[-\frac{s_{i}}{s_{i}^{2}-u_{j}^{2}}+\frac{1}{2 u_{j}} \ln \left|\frac{s_{i}+u_{j}}{s_{i}-u_{j}}\right|\right] \\
& +\eta_{4} \frac{1}{2 u_{j}} \ln \left|\frac{a+s_{i}}{a-s_{i}}\right|,
\end{aligned}
$$

$$
\begin{aligned}
D_{3}= & \sum_{i=1}^{N_{2}} \sum_{j=1}^{N_{1}} w_{j} \eta_{3}\left[-\frac{u_{j}}{u_{j}^{2}-s_{i}^{2}}+\frac{1}{2 s_{i}} \ln \left|\frac{u_{j}+s_{i}}{u_{j}-s_{i}}\right|\right] \\
& +\eta_{4} \frac{1}{2 s_{i}} \ln \left|\frac{a+u_{j}}{a-u_{j}}\right|, \\
D_{4}= & R \delta_{i j}+\sum_{i=1}^{N_{2}} \sum_{j=1}^{N_{2}} w_{j} \eta_{2} \frac{a}{s_{i} u_{j}},
\end{aligned}
$$

where $\delta_{i j}$ is the Kronecker delta, and $w_{j}$, as the weight function, is as follows:

$$
w_{j}= \begin{cases}\frac{a}{N_{1}}, & 0<r<a \\ t_{i+1}-t_{i}, & b<r<\infty\end{cases}
$$

The total load acting on the inclusion from Eq. (48) is:

$$
\begin{aligned}
\frac{T}{\delta}= & 8 a \eta_{4}+\frac{8 \gamma_{1} \eta_{3}}{\pi^{2}}\left[\sum_{i=1}^{N_{1}} w_{i} \phi_{1}\left(u_{i}\right) \ln \left|\frac{a+u_{i}}{a-u_{i}}\right|\right. \\
& \left.+\pi a \sum_{i=1}^{N_{2}} w_{i} \frac{\phi_{2}\left(u_{i}\right)}{u_{i}}\right] .
\end{aligned}
$$

It can be written in terms of axial stiffness which is defined as follows:

$$
\bar{T}=\frac{T}{c_{44} a \delta} .
$$

The stress intensity factor, defined by Eq. (52), can be expressed in the form of:

$$
K_{I I}^{b}=\frac{2 \delta \gamma_{1} \phi_{2}(b)}{\pi^{2} \sqrt{b}} .
$$

The normalized stress intensity factor can take the following form:

$$
\bar{K}_{I I}^{b}=\frac{K_{\mathrm{II}}^{b} b^{3 / 2}}{c_{44} a \delta} .
$$

\section{Numerical results and discussion}

To confirm the validity of the present solution and evaluate the effects of anisotropic materials on the results, several synthetic types of isotropic (material 1) and transversely isotropic materials (materials 2-9) are selected. The material properties are given in Table 1, where $E$ and $E^{\prime}$ are the Young's modules on the plane of isotropy and perpendicular to it, respectively; $\nu^{\prime}$ is Poisson's ratio that characterizes the effect of horizontal strain on the complementary vertical strain; $\nu$ is the Poisson's ratio which characterizes the effect of 
Table 1. Properties of synthetic materials.

\begin{tabular}{cccccccccccccc}
\hline Material & $\boldsymbol{E}$ & $\boldsymbol{E}^{\prime}$ & $\boldsymbol{G}$ & $\boldsymbol{G}^{\prime}$ & $\boldsymbol{E} / \boldsymbol{E}^{\prime}$ & $\boldsymbol{G} / \boldsymbol{G}^{\prime}$ & $\boldsymbol{\nu}, \boldsymbol{\nu}^{\prime}$ & $\boldsymbol{c}_{\mathbf{1 1}}$ & $\boldsymbol{c}_{\mathbf{1 2}}$ & $\boldsymbol{c}_{\mathbf{1 3}}$ & $\boldsymbol{c}_{\mathbf{3 3}}$ & $\boldsymbol{c}_{\mathbf{4 4}}$ & $\boldsymbol{c}_{\boldsymbol{6 6}}$ \\
\hline $1^{\mathrm{a}}$ & 5 & 5 & 2 & 2 & 1 & 1 & 0.25 & 6 & 2 & 2 & 6 & 2 & 2 \\
$2^{\mathrm{b}}$ & 5 & 10 & 2 & 2 & 0.5 & 1 & 0.25 & 5.6 & 1.6 & 1.8 & 10.9 & 2 & 2 \\
$3^{\mathrm{b}}$ & 5 & 25 & 2 & 2 & 0.2 & 1 & 0.25 & 5.4 & 1.4 & 1.7 & 25.9 & 2 & 2 \\
$4^{\mathrm{b}}$ & 5 & 5 & 2 & 1 & 1 & 2 & 0.25 & 6 & 2 & 2 & 6 & 1 & 2 \\
$5^{\mathrm{b}}$ & 5 & 5 & 2 & 0.67 & 1 & 3 & 0.25 & 6 & 2 & 2 & 6 & 0.67 & 2 \\
$6^{\mathrm{b}}$ & 5 & 5 & 2 & 0.4 & 1 & 5 & 0.25 & 6 & 2 & 2 & 6 & 0.4 & 2 \\
$7^{\mathrm{b}}$ & 10 & 5 & 4 & 2 & 2 & 2 & 0.25 & 14 & 6 & 5 & 7.5 & 2 & 4 \\
$8^{\mathrm{b}}$ & 15 & 5 & 6 & 2 & 3 & 3 & 0.25 & 26 & 14 & 10 & 10 & 2 & 6 \\
$9^{\mathrm{b}}$ & 25 & 5 & 10 & 2 & 5 & 5 & 0.25 & 110 & 90 & 50 & 30 & 2 & 10 \\
\hline
\end{tabular}

asotropic; ${ }^{\mathrm{b}}$ Transversely isotropic.

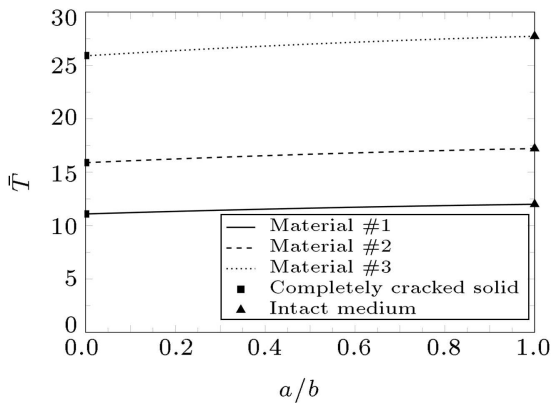

(a)

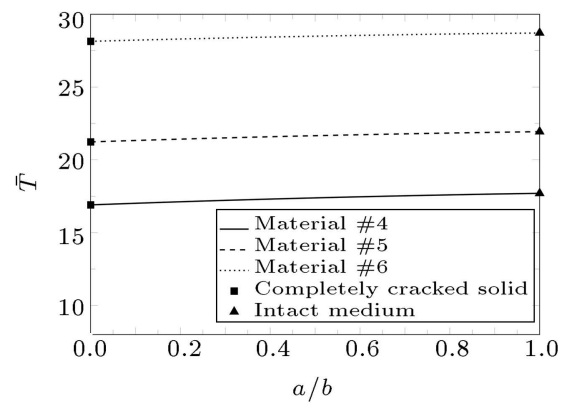

(b)

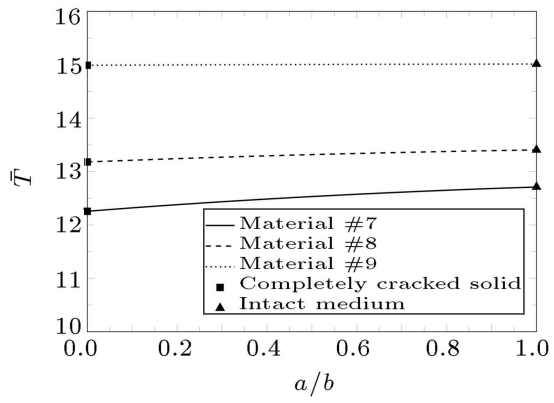

(c)

Figure 3. Axial stiffness for synthetic materials: (a) The effect of $E^{\prime}$, (b) the effect of $G^{\prime}$, and (c) the effects of $E$ and $G$.

vertical strain on the horizontal one; and $G^{\prime}$ stands for the shear modulus on the plane normal to the plane of isotropy. Regarding the positive-definiteness of strain energy, the following constraints for material constants $c_{i j}$ have been checked (for example, see [41])

$$
c_{11}>\left|c_{12}\right|, \quad\left(c_{11}+c_{12}\right) c_{33}>2 c_{13}^{2}, \quad c_{44}>0 .
$$

Axial stiffness as a function of the inclusion-crack aspect ratio is plotted in Figure 3 . The influence of $E^{\prime} / E$ ratio is shown in Figure 3(a), implying that an increase in $E^{\prime} / E$ leads to a remarkable increase in the axial stiffness. From Figure 3(b), one might notice that by decreasing $G^{\prime} / G$, the stiffness increases significantly. It can be concluded that anisotropic parameters $E^{\prime}$ and $G^{\prime}$ have the major influence on the axial stiffness. In contrast, from Figure 3(c), one can observe that the increase of $E / E^{\prime}$ and $G / G^{\prime}$ has little effect on the results. However, anisotropic parameters $E$ and $G$ are found to be of minor importance for the axial interaction of crack-inclusion.

The results of the synthetic transversely isotropic materials for the normalized shearing stress intensity factor are presented in Figure 4. As indicated in
Figure 4(a), the larger the value of $E^{\prime} / E$ is, the higher the response rate will be. Figure 4(b) shows that the reduction of $G^{\prime} / G$ leads to a slight increase in the mode II stress intensity factor. As shown in Figure 4(c), changing the ratios of $E / E^{\prime}$ and $G / G^{\prime}$ has a significant influence on $\sigma_{r z}$ and $K_{I I}^{b}$.

In the limiting $a \rightarrow b$, the normalized stress intensity factor decreases sharply, which is due to the oscillatory stress singularity. Selvadurai [12] showed that such oscillatory stress singularities have virtually no influence on the accuracy of the translational stiffness. In this study, $\bar{K}_{I I}^{b}$ is depicted for the interval $(0,0.9)$. Hilbert solution has been suggested to overcome this problem in the integral transform method.

\section{Conclusions}

The analytical treatment of the interaction crackinclusion in a transversely isotropic full-space was revisited. By virtue of appropriate potential functions, the mixed boundary value problem was reduced to dual and triple integral equations. By employing suitable representations and Abel transforms, the results were expressed in terms of the solution of two coupled Fred- 


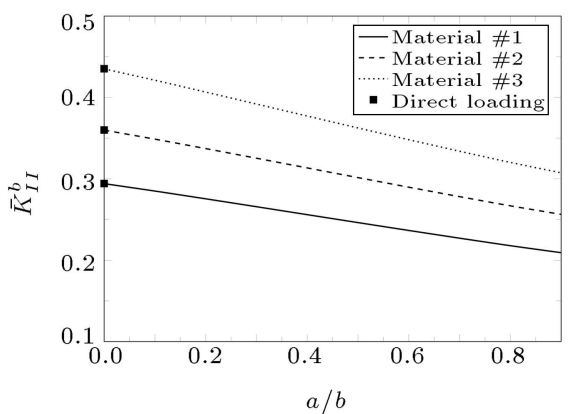

(a)

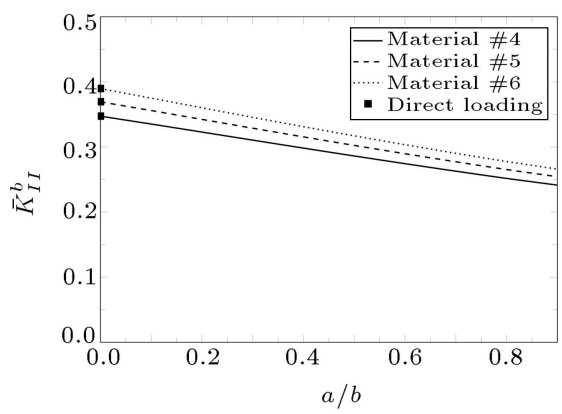

(b)

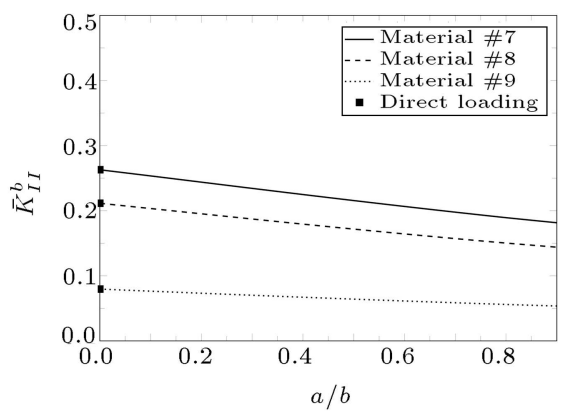

(c)

Figure 4. Normalized mode II stress intensity factor for synthetic materials: (a) The effect of $E^{\prime}$, (b) the effect of $G^{\prime}$, and (c) the effects of $E$ and $G$.

holm integral equations, solved by using a numerical method. The available closed-form results derived from the literature, such as the inclusion in an intact medium, were recovered as the limiting cases of the current study. The axial stiffness of the inclusion and the shearing stress intensity factor at the tip of the crack were obtained for some synthetic transversely isotropic materials. The effects of material anisotropy on the results were also highlighted.

\section{References}

1. Selvadurai, A.P.S. "Mechanics of a rigid circular disc bonded to a cracked elastic half-space", Int. J. Solids Struct., 39(24), pp. 6035-6053 (2002).

2. Menshykov, O.V., Menshykov, V.A., and Guz, I.A. "The contact problem for an open penny-shaped crack under normally incident tension-compression wave", Eng. Fract. Mech., 75(5), pp. 1114-1126 (2008).

3. Selvadurai, A.P.S. "A contact problem for a smooth rigid disc inclusion in a penny-shaped crack", $Z$. angew. Math. Phys. (ZAMP), 45(1), pp. 166-173 (1994).

4. Sneddon, I.N. "The distribution of stress in the neighbourhood of a crack in an elastic solid", Proc. Math. Phys. Eng. Sci., 187, pp. 229-260 (1946).

5. Collins, W. "Some axially symmetric stress distributions in elastic solids containing penny-shaped cracks. i. Cracks in an infinite solid and a thick plate", Proc. Math. Phys. Eng. Sci., 266, pp. 359-386 (1962).

6. Sneddon, I.N. and Tweed, J. "The stress intensity factor for a penny-shaped crack in an elastic body under the action of symmetric body forces", Int. J. Fract. Mech., 3(4), pp. 291-299 (1967).

7. Sneddon, I.N. and Lowengrub, M., Crack Problems in the Classical Theory of Elasticity, John Wiley \& Sons, 221 pages (1969).

8. Kassir, M. and Sih, G.C., Three-dimensional crack problems: A new selection of crack solutions in threedimensional elasticity, Noordhoof International Publishing, Leyden (1975).
9. Sih, G.C. "A review of the three-dimensional stress problem for a cracked plate", Int. J. Fract. Mech., 7(1), pp. 39-61 (1971).

10. Erdogan, F. and Arin, K. "Penny-shaped interface crack between an elastic layer and a half space", Int. J. Eng. Sci., 10(2), pp. 115-125 (1972).

11. Rahman, M. "A penny-shaped crack under timeharmonic torsional body forces", Eng. Fract. Mech., 49(4), pp. 599-610 (1994).

12. Selvadurai, A.P.S., Singh, B.M., and Au, M. "Axial loading of a rigid disc inclusion with a debonded region", Int. J. Solids Struct., 25(7), pp. 783-795 (1989).

13. Selvadurai, A.P.S. "On the axisymmetric loading of an annular crack by a disk inclusion", J. Eng. Math., 46(3-4), pp. 377-393 (2003).

14. Vrbik, J., Singh, B.M., Rokne, J., and Dhaliwal, R.S. "On the expansion of a penny-shaped crack by a rigid circular disc inclusion in a thick plate", Z. Angew. Math. Mech. (ZAMM), 84(8), pp. 538-550 (2004).

15. Eskandari, M., Moeini-Ardakani, S.S., and Shodja, H.M. "Axisymmetric contact of a rigid inclusion embedded at the interface of a piezoelectric bimaterial", Quart. J. Mech. Appl. Math., 62(3), pp. 281-295 (2009).

16. Shodja, H.M., Moeini-Ardakani, S.S., and Eskandari, M. "Axisymmetric problem of energetically consistent interacting annular and penny-shaped cracks in piezoelectric materials", J. Appl. Mech., 78(2), pp. 02101010 (2011).

17. Eskandari, M., Moeini-Ardakani, S.S., and Shodja, H.M. "An energetically consistent annular crack in a piezoelectric medium", Eng. Fract. Mech, 77(5), pp. 819-831 (2010).

18. Singh, B.M., Rokne, J., and Dhaliwal, R.S. "Closed form solution for an annular elliptic crack around an elliptic rigid inclusion in an infinite solid", J. Appl. Math. Mech. (ZAMM), 92(11-12), pp. 882-887 (2012).

19. Eskandari-Ghadi, M., Ardeshir-Behrestaghi, A., and Neya, B.N. "Mathematical analysis for an axisymmetric disc-shaped crack in transversely isotropic halfspace", Int. J. Mech. Sci., 68, pp. 171-179 (2013). 
20. Fabrikant, V.I. "General flat crack located in the plane perpendicular to the planes of isotropy in transversely isotropic body", Acta Mech., 226(10), pp. 3289-3306 (2015).

21. Fabrikant, V.I. "Non-traditional crack problem for transversely-isotropic body", Eur. J. Mech. A-Solid, 30(6), pp. 902-912 (2011).

22. Fabrikant, V.I. "General flat crack arbitrarily located in the transversely-isotropic body", Theor. Appl. Fract. Mech., 82, pp. 69-76 (2016).

23. Fabrikant, V. "Relationship between contact and crack problems for generally anisotropic bodies", Int. J. Eng. Sci., 102, pp. 27-35 (2016).

24. Yang, F. and Zhao, Y.-P. "The effect of a capillary bridge on the crack opening of a penny crack", Soft Matter, 12(5), pp. 1586-1592 (2016).

25. Shahmohamadi, M., Khojasteh, A., and Rahimian, M. "Frictionless contact of a rigid disk with the face of a penny-shaped crack in a transversely isotropic solid", Int. J. Solids Struct., 106, pp. 274-283 (2017).

26. Antipov, Y.A. and Mkhitaryan, S.M. "A crack induced by a thin rigid inclusion partly debonded from the matrix", Quart. J. Mech. Appl. Math., 70(2), pp. 15385 (2017).

27. Amiri-Hezaveh, A., Moghaddasi, H., Karimi, P., and Ostoja-Starzewski, M. "Dynamic interaction of plates in an inhomogeneous transversely isotropic space weakened by a crack", J. Appl. Math. Mech. (ZAMM), 97, pp. 491-504 (2017).

28. Eskandari-Ghadi, M., Ardeshir-Behrestaghi, A., and Pak, R.Y. "Bi-material transversely isotropic halfspace containing penny-shaped crack under timeharmonic horizontal loads", Eng. Fract. Mech., 172, pp. 152-180 (2017).

29. Singh, B.M., Rokne, J., and Dhaliwal, R.S. "Thermal stresses in a two-dimensional infinite medium containing a rigid inclusion embedded in a line crack", Theor. Appl. Fract. Mech., 46(2), pp. 148-155 (2006).

30. Lekhnitskii, S., Theory of an Anisotropic Elastic Body, 525. Holden-Day, San Francisco (1963).

31. Rahimian, M., Eskandari-Ghadi, M., Pak, R.Y.S., and Khojasteh, A. "Elastodynamic potential method for transversely isotropic solid", J. Eng. Mech., 133(10), pp. 1134-1145 (2007).

32. Cooke, J. "Triple integral equations", Quart. J. Mech. Appl. Math., 16(2), pp. 193-203 (1963).

33. Katebi, A.A., Khojasteh, A., Rahimian, M., and Pak, R.Y.S. "Axisymmetric interaction of a rigid disc with a transversely isotropic half-space", Int. J. Numer. Anal. Methods Geomech., 34(12), pp. 1211-1236 (2010).

34. Selvadurai, A.P.S. "Axial displacement of a rigid elliptical disc inclusion embedded in a transversely isotropic elastic solid", Mech. Res. Commun., 9(1), pp. 39-45 (1982).
35. Fabrikant, V.I., Mixed Boundary Value Problems of Potential Theory and Their Applications in Engineering, Kluwer Academic Publishers (1991).

36. Mossakovskii, V. "The fundamental mixed problem of the theory of elasticity for a half-space with a circular line separating the boundary conditions", Prikl. Mat. Mekh., 18, pp. 187-196 (1954).

37. Fabrikant, V.I., Applications of Potential Theory in Mechanics: a Selection of New Results, 51, Kluwer Academic Publishers, Dordrecht, Boston (1989).

38. Selvadurai, A.P.S. and Singh, B.M. "Axisymmetric problems for an externally cracked elastic solid-ii. Effect of a penny-shaped inclusion", Int. J. Eng. Sci., 25(11-12), pp. 1477-1490 (1987).

39. Baker, C., The Numerical Treatment of Integral Equations, Clarendon Press (1977).

40. Atkinson, K.E. A Survey of Numerical Methods for the Solution of Fredholm Integral Equations of the Second Kind, Soc. for Industrial and Applied Mathematics (1976).

41. Payton, R.C., Elastic Wave Propagation in Transversely Isotropic Media, Springer Science \& Business Media (2012).

\section{Appendix A}

The parameters in Eqs. (10)-(13) are:

$$
\begin{aligned}
& \gamma_{1}=-\frac{C_{2}}{C_{1}}, \\
& \gamma_{2}=-\frac{C_{3}}{C_{4}}, \\
& C_{1}=-\frac{1+\alpha_{1}+s_{1} s_{2} \alpha_{2}}{c_{44}\left(s_{1}+s_{2}\right)\left(1+\alpha_{1}\right)}, \\
& C_{2}=\frac{-1-\alpha_{1}+s_{1} s_{2}\left(\alpha_{3}-\alpha_{2}\right)}{\left(s_{1}+s_{2}\right)\left(1+\alpha_{1}\right)} .
\end{aligned}
$$

Eqs. (A.5) to (A.8) are shown in Box A.I.

\section{Appendix B}

The parameters in Eqs. (55) and (57) are:

$$
\begin{aligned}
& m_{k}=\frac{c_{13}+c_{44}}{c_{33} s_{k}^{2}-c_{44}} \quad(k=1,2), \\
& \theta=\frac{1}{2 \pi} \ln \left[\frac{\sqrt{\gamma_{1}^{\prime} \gamma_{2}^{\prime}}+\alpha}{\sqrt{\gamma_{1}^{\prime} \gamma_{2}^{\prime}}-\alpha}\right], \\
& H=\frac{\left(\gamma_{1}^{\prime}+\gamma_{2}^{\prime}\right) c_{11}}{2 \pi\left(c_{11} c_{33}-c_{13}^{2}\right)}, \\
& \alpha=\frac{\sqrt{c_{11} c_{33}}-c_{13}}{c_{11}\left(\gamma_{1}^{\prime}+\gamma_{2}^{\prime}\right)}, \\
& \gamma_{k}^{\prime}=s_{k}^{-1}=\sqrt{\frac{m_{k} c_{33}}{m_{k} c_{44}+c_{13}+c_{44}}} \quad(k=1,2) .
\end{aligned}
$$




$$
\begin{aligned}
& C_{3}=\frac{c_{33}\left(1+\alpha_{1}-s_{1}^{2} \alpha_{2}\right)\left(1+\alpha_{1}-s_{2}^{2} \alpha_{2}\right)-c_{13}\left(1+\alpha_{1}+s_{1} s_{2} \alpha_{2}\right) \alpha_{3}}{c_{44}\left(s_{1}+s_{2}\right)\left(1+\alpha_{1}\right) \alpha_{3}} \\
& C_{4}=\frac{c_{33}\left(1+\alpha_{1}-s_{1}^{2} \alpha_{2}\right)\left(1+\alpha_{1}-s_{2}^{2} \alpha_{2}\right)-\left(c_{13}+c_{33} s_{1} s_{2}\right)\left(1+\alpha_{1}+s_{1} s_{2} \alpha_{2}\right) \alpha_{3}+c_{13} s_{1} s_{2} \alpha_{3}^{2}}{\left(s_{1}+s_{2}\right)\left(1+\alpha_{1}\right) \alpha_{3}} \\
& A(\xi)=\frac{\left(N(\xi)+M(\xi) c_{44}\right)\left(1+\alpha_{1}-s_{2}^{2} \alpha_{2}\right)+M(\xi) c_{44} s_{2}^{2} \alpha_{3}}{\xi c_{44}\left(s_{1}^{2}-s_{2}^{2}\right)\left(1+\alpha_{1}\right) \alpha_{3}} \\
& B(\xi)=-\frac{\left(N(\xi)+M(\xi) c_{44}\right)\left(1+\alpha_{1}-s_{1}^{2} \alpha_{2}\right)+M(\xi) c_{44} s_{1}^{2} \alpha_{3}}{\xi c_{44}\left(s_{1}^{2}-s_{2}^{2}\right)\left(1+\alpha_{1}\right) \alpha_{3}}
\end{aligned}
$$

Box A.I

$$
\begin{aligned}
& u_{z}(r, z)=\int_{0}^{\infty} \frac{\xi z \kappa_{1}\left(N(\xi)+M(\xi) c_{44}\right)+M(\xi) \omega_{1}(\xi) c_{44} s_{1} \alpha_{3}}{2 c_{44} s_{1}\left(1+\alpha_{1}\right) \alpha_{3}} \mathrm{e}^{-\xi s_{1} z} J_{0}(\xi r) \mathrm{d} \xi \\
& u_{r}(r, z)=\int_{0}^{\infty} \frac{\left(N(\xi)+M(\xi) c_{44}\right) \omega_{2}(\xi)+M(\xi) c_{44} s_{1}^{2} \alpha_{3}\left(1+\xi s_{1} z\right)}{2 c_{44} s_{1}\left(1+\alpha_{1}\right)} \mathrm{e}^{-\xi s_{1} z} J_{1}(\xi r) \mathrm{d} \xi \\
& \sigma_{z z}(r, z)=-\int_{0}^{\infty} \frac{c_{13} \alpha_{3} \Gamma_{1}(\xi)+c_{33} \Gamma_{2}(\xi)}{2 c_{44} s_{1}\left(1+\alpha_{1}\right) \alpha_{3}} \xi \mathrm{e}^{-\xi s_{1} z} \mathrm{~d} \xi \\
& \sigma_{r z}(r, z)=-\int_{0}^{\infty} \frac{\xi z \kappa_{1} N(\xi)+\alpha_{3} s_{1} \omega_{3}(\xi) N(\xi)+c_{44} \xi z \kappa_{2} M(\xi)}{2 s_{1}\left(1+\alpha_{1}\right) \alpha_{3}} \xi \mathrm{e}^{-\xi s_{1} z} J_{1}(\xi r) \mathrm{d} \xi
\end{aligned}
$$

Box C.I

\section{Appendix C}

Displacement and stress potential relations of the case of $s_{1}=s_{2}$ are shown in Box C.I, where:

$$
\begin{aligned}
& \kappa_{1}=\left(1+\alpha_{1}-s_{1}^{2} \alpha_{2}\right)^{2} \\
& \kappa_{2}=\left(1+\alpha_{1}+s_{1}^{2}\left(\alpha_{3}-\alpha_{2}\right)\right)^{2} \\
& \omega_{1}(\xi)=\left(2+\xi s_{1} z\right)\left(1+\alpha_{1}\right)-\xi s_{1}^{3} \alpha_{2} z \\
& \omega_{2}(\xi)=\left(\xi s_{1} z-1\right)\left(1+\alpha_{1}\right)-s_{1}^{2} \alpha_{2}\left(1+z \xi s_{1}\right), \\
& \omega_{3}(\xi)=\left(\xi s_{1} z-2\right)\left(1+\alpha_{1}\right)-\xi s_{1}^{3} \alpha_{2} z \\
& \omega_{4}(\xi)=1+\xi s_{1} z \\
& \omega_{5}(\xi)=1-\xi s_{1} z \\
& \Gamma_{1}(\xi)=\omega_{5}(\xi)\left(N(\xi)+M(\xi) c_{44}\right)
\end{aligned}
$$$$
\left(\left(1+\alpha_{1}\right)+s_{1}^{2} \alpha_{2}\right)-\omega_{4}(\xi) M(\xi) c_{44} \alpha_{3} s_{1}^{2}
$$

$$
\begin{aligned}
\Gamma_{2}(\xi)= & -\omega_{5}(\xi) \kappa_{1}\left(N(\xi)+M(\xi) c_{44}\right) \\
& +M(\xi) c_{44} \alpha_{3} s_{1}^{2}\left(\omega_{4}(\xi)\left(1+\alpha_{1}\right)\right. \\
& \left.+\omega_{5}(\xi) s_{1}^{2} \alpha_{2}\right) .
\end{aligned}
$$

\section{Biographies}

Seyyed Morteza Dehghan Manshadi received his BSc degree in Civil Engineering from Yazd University in 2013. He received his MSc degree in Structural Engineering from University of Tehran in 2017. Solid mechanics and fracture mechanics are among his main research interests.

Ali Khojasteh, currently Assistant Professor at the University of Tehran, received his BSc in Civil Engineering from the Shiraz University, Iran, in 2003 and MSc and PhD degrees from the University of Tehran, Iran, in 2005 and 2009, respectively, both ranked 1st among graduates. His research interests are computational mechanics, wave propagation in solids/fluids, mechanics of composites, BEM, soil-structure Interaction. He was ranked 262nd among more than 800,000, 
7th among more than 15000 and 9th among more than 15000 participating in the nationwide undergraduate and MSc program entrance exams and the Civil Engineering Olympiad, respectively. More than 25 papers published in ISI indexed journals, are of his scientific works.

Mohammad Rahimian, currently the Full Professor of Civil Engineering Department at the University of Tehran, received his BSc in Civil Engineering from the
University of Tehran. He received his $\mathrm{PhD}$ in Ecole National Des Ponts et Chaussees, France, in 1981. His research fields are earthquake engineering, wave propagation in anisotropic materials, soil-structurefluid interaction, etc. $\mathrm{He}$ is the former chair and assistant to chair of the University of Tehran and the former chair of the Faculty of Engineering of the University of Tehran. More than 60 papers published in ISI indexed journals, and 6 written or translated books are of his scientific works. 